\title{
Stereotactic Body Radiation Therapy as an Alternative Treatment for Small Hepatocellular Carcinoma
}

\author{
Sang Min Yoon ${ }^{1}$, Young-Suk Lim², Mee Jin Park³, So Yeon Kim³, Byungchul Cho', Ju Hyun Shim², Kang \\ Mo Kim², Han Chu Lee ${ }^{2}$, Young-Hwa Chung ${ }^{2}$, Yung Sang Lee ${ }^{2}$, Sung Gyu Lee ${ }^{4}$, Yu Sun Lee ${ }^{1}$, Jin-hong \\ Park ${ }^{1}$, Jong Hoon Kim ${ }^{1 *}$
}

1 Department of Radiation Oncology, Asan Medical Center, University of Ulsan College of Medicine, Songpa-gu, Seoul, Republic of Korea, 2 Department of Gastroenterology, Asan Medical Center, University of Ulsan College of Medicine, Songpa-gu, Seoul, Republic of Korea, 3 Department of Radiology, Asan Medical Center, University of Ulsan College of Medicine, Songpa-gu, Seoul, Republic of Korea, 4 Department of Surgery, Asan Medical Center, University of Ulsan College of Medicine, Songpa-gu, Seoul, Republic of Korea

\begin{abstract}
Background: Even with early stage hepatocellular carcinoma (HCC), patients are often ineligible for surgical resection, transplantation, or local ablation due to advanced cirrhosis, donor shortage, or difficult location. Stereotactic body radiation therapy (SBRT) has been established as a standard treatment option for patients with stage I lung cancer, who are not eligible for surgery, and may be a promising alternative treatment for patients with small HCC who are not eligible for curative treatment.

Materials and Methods: A registry database of 93 patients who were treated with SBRT for HCC between 2007 and 2009 was analyzed. A dose of 10-20 Gy per fraction was given over 3-4 consecutive days, resulting in a total dose of 30-60 Gy. The tumor response was determined using dynamic computed tomography or magnetic resonance imaging, which was performed 3 months after completion of SBRT.

Results: The median follow-up period was 25.6 months. Median size of tumors was $2 \mathrm{~cm}$ (range: $1-6 \mathrm{~cm}$ ). Overall patients' survival rates at 1 and 3 years were $86.0 \%$ and $53.8 \%$, respectively. Complete and partial tumor response were achieved in $15.5 \%$ and $45.7 \%$ of patients, respectively. Local recurrence-free survival rate was $92.1 \%$ at 3 years. Most local failures were found in patients with $\mathrm{HCCs}>3 \mathrm{~cm}$, and local control rate at 3 years was $76.3 \%$ in patients with $\mathrm{HCC}>3 \mathrm{~cm}, 93.3 \%$ in patients with tumors between $2.1-3 \mathrm{~cm}$, and $100 \%$ in patients with tumors $\leq 2$ $\mathrm{cm}$, respectively. Out-of-field intrahepatic recurrence-free survival rates at 1 and 3 years were $51.9 \%$ and $32.4 \%$, respectively. Grade $\geq 3$ hepatic toxicity was observed in $6(6.5 \%)$.
\end{abstract}

Conclusions: SBRT was effective in local control of small HCC. SBRT may be a promising alternative treatment for patients with small HCC which is unsuitable for other curative therapy.

Citation: Yoon SM, Lim Y-S, Park MJ, Kim SY, Cho B, et al. (2013) Stereotactic Body Radiation Therapy as an Alternative Treatment for Small Hepatocellular Carcinoma. PLoS ONE 8(11): e79854. doi:10.1371/journal.pone.0079854

Editor: Tetsuo Takehara, Osaka University Graduate School of Medicine, Japan

Received June 20, 2013; Accepted September 25, 2013; Published November 8, 2013

Copyright: @ 2013 Yoon et al. This is an open-access article distributed under the terms of the Creative Commons Attribution License, which permits unrestricted use, distribution, and reproduction in any medium, provided the original author and source are credited.

Funding: The authors have no funding or support to report.

Competing interests: The authors have declared that no competing interests exist.

*E-mail: jhkim2@amc.seoul.kr

\section{Introduction}

Hepatocellular carcinoma ( $\mathrm{HCC}$ ) is the third most common cause of death from cancer worldwide [1]. Current practice guidelines recommend hepatic resection, liver transplantation, and percutaneous ablation as curative treatment options [2]. However, hepatic resection can only be offered to $10 \%$ to $30 \%$ of patients at diagnosis because of various clinical reasons [3], and the use of liver transplantation is also very limited due to the lack of donors and stringent indications. Although percutaneous ablative therapy, including radiofrequency ablation (RFA) and percutaneous ethanol injection (PEI), can also be used with curative intent for the treatment of small HCC which is unsuitable for surgery, this ablative therapy cannot be safely performed when HCC lesions are positioned at deep locations, near to the bile duct or large vessel, at the top of the dome, or are undetectable by ultrasound. Therefore, alternative non-invasive local therapeutic modalities are indispensable in these clinical settings.

Recent improvements in radiotherapy techniques, including the conformal delivery of radiation, techniques that account for respiratory motion, image-guided radiotherapy (IGRT), and information on partial volume liver tolerance, allow the delivery of higher doses of radiation to these tumors than previously 
thought possible, thereby allowing radiotherapy to be used as an alternative option for treating HCC [4]. Of the available radiotherapy options, stereotactic body radiation therapy (SBRT) has emerged as a non-invasive local treatment option for patients with HCC when established curative treatment modalities cannot be applied. Although prospective studies remain sparse at this time, many previous clinical studies have reported SBRT to be safe and efficacious for the treatment of HCC [5-8].

In our current study, we report our clinical experiences with SBRT as an alternative treatment for small, unresectable HCC and evaluate the long-term efficacy and safety of this highly sophisticated treatment modality.

\section{Materials and Methods}

\section{Ethics statement}

This study was approved by the Institutional Review Board of the Asan Medical Center, and informed consent in writing was obtained from each patient in the study.

\section{Patients}

Patients who were treated with SBRT for primary or recurrent $\mathrm{HCC}$ were registered and the database was retrospectively reviewed between March 2007 and December 2009. Eligibility criteria for the present study included the following: (1) the HCC lesion was not suitable for surgery due to liver cirrhosis, no sufficient remnant liver for resection after previous surgery, and patients' refusal of surgery; (2) the HCC was located in liver surface, near to the bile duct or large vessels, or at the top of the dome where percutaneous ablative therapies cannot be safely performed; (3) the HCC was confined to the liver without extrahepatic metastases; (4) the HCC was $<6 \mathrm{~cm}$ across its longest diameter, and $\leq 3$ lesions were present; (5) the HCC demonstrated no evidence of major vascular invasions; (6) liver function was classified as Child-Pugh class A or B; (7) an adequate residual functional liver volume was evident $(>700$ $\mathrm{cc})$; (8) there was a sufficient distance $(>2 \mathrm{~cm}$ ) between the HCC and adjacent organs at risk, such as the duodenum, stomach, colon, and spinal cord; (9) an incomplete response after transarterial chemoembolization (TACE) or unsuitable for TACE due to the lesion non-visibility on hepatic angiogram; and (10) no prior history of external beam radiotherapy.

In all of our patients, the diagnosis of HCC was based on (1) histological confirmation; or (2) a characteristic tumor appearance by at least two imaging studies (including dynamic computed tomography (CT) scans, dynamic enhanced magnetic resonance imaging (MRI) scans and angiograms); and (3) the presence of risk factors, including hepatitis B virus, hepatitis $C$ virus infection and cirrhosis.

\section{Simulation and target volume delineations}

At least one week before CT simulation, we implanted three gold seeds (CIVCO Medical Solutions, Kalona, IA, USA) into the liver parenchyma around the tumor under sonographic guidance in almost every patient. The gold seeds were not implanted in some patients who had surgical clips or compact iodized oil, which still remained after previous treatments. All patients were immobilized in the supine position using a vacuum cushion, and while freely breathing 4-dimensional (4D) CT scanning was performed using 16-slice CT system (GE LightSpeed RT 16; GE Healthcare, Waukesha, WI, USA). A Real-time Position Management respiratory gating system was used to record the patients' breathing patterns (Varian Medical Systems, Palo Alto, CA, USA). All CT datasets were sorted into 10 phase bins that corresponded to the respiratory phase using 4D imaging software (Advantage 4D; GE Healthcare).

The gross tumor volume (GTV), as determined by dynamic enhanced CT or MRI, included an enhanced mass at the endexpiratory phase of the CT image (50\% phase). No GTV to clinical target volume margin was added for taking into account subclinical extension. The internal target volume (ITV) was delineated as the sum of the individual GTVs, as defined within the gated phases of respiration (usually $30-70 \%$ phase). The planning target volume (PTV) was expanded to include a 0.5 $\mathrm{cm}$ margin from the ITV. The whole and normal liver, both kidneys, spinal cord, duodenum, and stomach were delineated and 3- dimensionally reconstructed.

\section{Treatment planning and delivery}

SBRT planning was performed using a 3-dimensional radiotherapy planning system (Eclipse; Varian Medical Systems) that used multiple static conformal beams with energies of 6- or 15-MV photons. A dose of 10-20 Gy (median: $15 \mathrm{~Gy}$ ) per fraction was given over 3-4 consecutive days, resulting in a total dose of 30-60 Gy (median: $45 \mathrm{~Gy}$ ) being administered to the isodose line covering the PTV. The total dose was mainly determined based on general dosing guidelines after determining the dose to be administered to the normal liver, including the following: (1) the maximum dose allowed to $700 \mathrm{cc}$ of normal liver was estimated to be $15 \mathrm{~Gy}$ in three fractions: and (2) the mean dose administered to normal liver was not to exceed $13 \mathrm{~Gy}$ in three fractions [9,10]. Dose limitations to other critical structures included the following: 2 cc of the esophagus or large bowel were to be limited to a total dose of $<21 \mathrm{~Gy}, 2 \mathrm{cc}$ of the stomach or duodenum were to be limited to a total dose of $<18 \mathrm{~Gy}$, and $2 \mathrm{cc}$ of the spinal cord were to be limited to a total dose of $18 \mathrm{~Gy}$.

Image guidance was performed in two stages before administering each fraction of SBRT using On-Board Imager (Varian Medical Systems). First, cone-beam CT was done and 3D matching was performed. Second, gated fluoroscopy was performed in the anterior-posterior and lateral directions to confirm the marker positions at the end-exhale phase.

\section{Evaluation and statistics}

All patients were examined during SBRT to assess acute toxicity. After treatment, regular follow-up examinations were performed at 2-3 month intervals. A review of each patient's prior medical history, physical examinations, complete blood counts, biochemical profiles, tumor markers, and imaging studies were performed at each follow-up. Adverse effects related to SBRT were graded according to the Common Terminology Criteria for Adverse Events (CTCAE; version 3.0). Radiation-induced hepatic toxicity was also graded according 
to CTCAE or any decline in liver function using Child-Pugh score in the absence of documented progressive disease within three months after SBRT.

Tumor response was defined at three months after the completion of SBRT according to both the Response Evaluation Criteria in Solid Tumors criteria (RECIST version 1.1) and the modified RECIST with the consensus of two radiologists (KSY and $\mathrm{PMJ}$ ). Local failure was defined as the recurrence of the treated lesion, intrahepatic recurrence was defined as recurrence within the liver outside the treated lesion, and extrahepatic metastasis was defined as recurrent disease at any site outside the liver. For local recurrence, the presence of wash-out on the portal and delayed phase images or increase in volume within the irradiated hepatic parenchyma was considered as such.

Overall and recurrence-free survivals were estimated from the date of the start of SBRT to the date of death, the last follow-up examination, or to the date of tumor recurrence, respectively. The probability of cumulative survival was calculated using the Kaplan-Meier method. Correlation between tumor sizes and radiation doses was performed using Spearman correlation analysis. In addition, the existence of a variable-effect relationship was confirmed by logistic regression analysis. These analyses were performed using SPSS (version 12.0; SPSS Inc., Chicago, IL, USA).

\section{Results}

\section{Patient characteristics}

A total of 129 patients with HCC who were treated with SBRT were registered between March 2007 and December 2009 at our institution. Among these, 28 patients were not included in our current analysis for the various reasons in Figure 1. The remaining 93 patients (103 lesions) met all of the enrollment criteria and were included in this analysis (Table 1). The study population was mostly male $(80.6 \%)$, demonstrating a median age of 61 years (range: $42-86$ years). Sixty-nine patients had liver function of Child-Pugh class A, and median tumor size was $2 \mathrm{~cm}$. Eighty-nine (95.7\%) patients were identified to have liver cirrhosis. Only 1 patient was treatmentnaïve, and all other patients had received various courses of previous therapies, including hepatic resection, TACE, RFA, or PEI before receiving SBRT. However, additional locoregional treatments were not performed on recurrent or residual viable HCCs if salvage SBRT was considered for the lesion. Before CT simulation, 88 patients $(94.6 \%)$ were implanted with 3 gold seeds to serve as fiducial markers for IGRT.

\section{Overall survival and tumor response}

The median follow-up period for all patients was 25.6 months (range: 1.8-55.4 months). At the time of analysis, 51 patients were alive and 42 patients were deceased. The causes of death were as follows: intrahepatic or extrahepatic tumor progression in 29 , progression of liver cirrhosis in 8 , other comorbidity in 2, complications after the following treatment in 1 , and unknown cause(s) in 2. The 1 and 3 years overall survival rates were $86.0 \%$ and $53.8 \%$, respectively (Figure 2 ).
CT or MRI at 3 months after SBRT was evaluated in 91 patients (101 lesions). Of these, $16(15.5 \%)$ achieved complete response, $47(45.7 \%)$ achieved partial response, and 38 $(36.9 \%)$ achieved stable disease, yielding an objective response rate of $61.2 \%$ according to the RECIST criteria (Table 2). Using the modified RECIST criteria, however, $53(51.5 \%)$ achieved complete response, 22 (21.4\%) achieved partial response, and $26(25.2 \%)$ achieved stable disease, yielding an objective response rate of $72.9 \%$ (Table 2). No progressive disease was observed in the treated HCCs.

\section{Patterns of failure and recurrence after SBRT}

Seven local failures were recorded at the time of analysis. Intrahepatic (i.e., out-of-field) recurrence was the main cause of failure (58 of 92 patients), and distant metastasis developed in 20 patients during the follow-up periods. Local control rates at 1 and 3 years were $94.8 \%$ and $92.1 \%$, respectively (Figure $3 \mathrm{~A}$ ), and distant metastasis-free survival rates at 1 and 3 years were $87.9 \%$ and $72.2 \%$, respectively (Figure 3B). However, intrahepatic recurrence-free survival rates at 1 and 3 years were $51.9 \%$ and $32.4 \%$, respectively (Figure $3 \mathrm{C}$ )

The incidence of local failure was significantly related to tumor size. Most local failures were found in patients with HCCs $>3 \mathrm{~cm}$, and local control rate at 3 years was $76.3 \%$ in patients with $\mathrm{HCC}>3 \mathrm{~cm}, 93.3 \%$ in patients with tumors between $2.1-3 \mathrm{~cm}$, and $100 \%$ in patients with tumors $\leq 2 \mathrm{~cm}$, respectively $(p=0.001)$ (Figure 4$)$. There was no significant correlation between tumor sizes and radiation doses $(p=0.078)$. Logistic regression analysis also revealed that the tumor size was the only significant factor determining the local tumor control $(p=0.001)$. Other variables, such as the age, Child-Pugh score, pretreatment alpha-fetoprotein level, and radiation dose were not significant factors.

\section{Additional treatment after SBRT for New Recurrent Lesions}

After the diagnosis of new recurrences, 65 patients received various types of additional treatments, including locoregional treatments for intrahepatic recurrences (e.g., segmentectomy, TACE, PEI, RFA, SBRT) or treatments for distant metastases (e.g., sorafenib, metastatectomy, SBRT for metastatic lesions, palliative external beam radiotherapy). Liver transplantation was performed in 7 patients. Of these, 4 patients received transplantation for the treatment of liver cirrhosis without tumor recurrence and 3 patients received transplantation in combination with other loco-regional treatments after intrahepatic recurrence.

\section{Treatment-related toxicity}

Symptomatic complications, including septic shock, due to the insertion of the gold seeds occurred in 1 patient. However, this individual recovered well before the start of SBRT with supportive care. All patients received the planned SBRT regimen with no interruptions due to intolerable side effects. Fatigue and anorexia were the most common acute toxicities, but these were mostly CTCAE grade 1 . Grade $\geq 3$ hepatic toxicities, without progression of intrahepatic $\mathrm{HCC}$, which may have been related to radiation, were observed in $6(6.5 \%)$ 


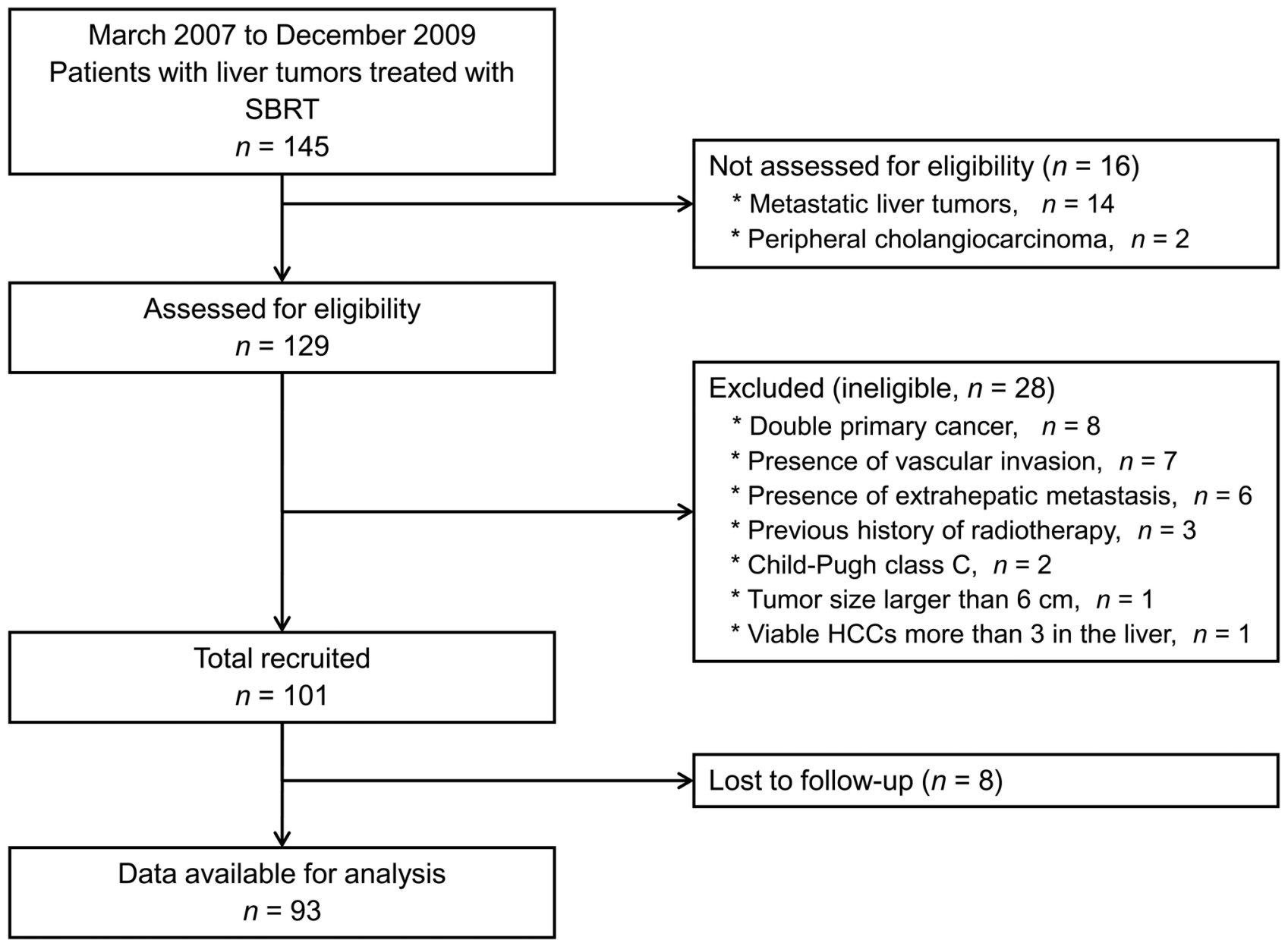

Figure 1. Flow diagram of this study.

doi: 10.1371/journal.pone.0079854.g001

patients within 3 months of SBRT and are summarized in Table 3. The main patterns of hepatic toxicities were elevations in serum liver enzyme levels or bilirubin levels. Nine (9.7\%) patients also experienced an elevation in the Child-Pugh score to $>2$ within 3 months of SBRT. Of these, one patient, who experienced grade 4 hyperbilirubinemia, died due to hepatic failure at 2 months after the completion of SBRT. However, it was difficult to differentiate the cause of hepatic failure from SBRT or other unrevealed reasons, because the treated tumor was small $(1.5 \mathrm{~cm})$, also located in the liver dome area, and the liver function was good (Child-Pugh class $A$ ). The remainder of the patients showed improved or stable liver function during the follow-up period. Both the pretreatment Child-Pugh class and the radiation dose were not correlated with the grade $\geq 3$ hepatic toxicities in logistic regression analyses $(p=0.081$ and $p=0.153$, respectively). There was no gastrointestinal complication such as bleeding or perforation, during the followup periods. Two patients developed rib fractures that did not require any specific treatment at 12 months and 18 months after SBRT, respectively. In these cases, the PTV included a portion of the fractured ribs. The total dose to those ribs was 30
Gy and 45 Gy, in three fractions, respectively. Biliary stricture developed in 1 patient, whose tumor was located in the central liver (segment 4), at 26 months after SBRT.

\section{Discussion}

Hepatic resection is the primary curative treatment for HCC. Due to advances in surgical techniques and improvements in postoperative management, the 5-year survival rate has increased to about $70 \%$, especially for small HCCs $<5 \mathrm{~cm}$ in diameter [11]. Unfortunately, only a small proportion of patients with HCC can undergo hepatic resection. For patients with early-stage HCC who are not suitable for surgery, percutaneous ablative therapy is another curative treatment option. Although, randomized trials comparing hepatic resection and RFA for the treatment of HCC reach different conclusions [12-14], RFA can achieve satisfactory local control rates and similar survival outcomes compared with hepatic resection, demonstrating 5-year survival rates of $50-75 \%$ in patients with liver function that is classified as Child-Pugh class A [15]. Based on these clinical outcomes, the recently updated 
Table 1. Patient characteristics.

\begin{tabular}{|c|c|}
\hline Variables & No. of patients (\%) \\
\hline \multicolumn{2}{|l|}{ Gender } \\
\hline Male & $75(80.6)$ \\
\hline Female & $18(19.4)$ \\
\hline \multicolumn{2}{|l|}{ Age (years) } \\
\hline Median & 61 \\
\hline Range & $42-86$ \\
\hline \multicolumn{2}{|l|}{ Child-Pugh class } \\
\hline A & $69(74.2)$ \\
\hline B & $24(25.8)$ \\
\hline \multicolumn{2}{|l|}{ Viral etiology } \\
\hline HBV & $69(74.2)$ \\
\hline $\mathrm{HCV}$ & 12 (12.9) \\
\hline Others & $12(12.9)$ \\
\hline \multicolumn{2}{|l|}{ Liver cirrhosis } \\
\hline Yes & $89(95.7)$ \\
\hline No & $4(4.3)$ \\
\hline \multicolumn{2}{|l|}{ Tumor size } \\
\hline $1.0-2.0 \mathrm{~cm}$ & $54(52.4)$ \\
\hline $2.1-3.0 \mathrm{~cm}$ & $18(17.5)$ \\
\hline $3.1-4.0 \mathrm{~cm}$ & $22(21.4)$ \\
\hline $4.1-5.1 \mathrm{~cm}$ & $6(5.8)$ \\
\hline $5.1-6.0 \mathrm{~cm}$ & $3(2.9)$ \\
\hline \multicolumn{2}{|c|}{ Number of viable tumors before SBRT } \\
\hline 1 & $83(89.2)$ \\
\hline 2 & $10(10.8)$ \\
\hline \multicolumn{2}{|l|}{ Alpha-fetoprotein (ng/mL) } \\
\hline Range & $0.8-2490$ \\
\hline$\leq 200$ & $78(83.9)$ \\
\hline$>200$ & $15(16.1)$ \\
\hline \multicolumn{2}{|l|}{ Prior treatments } \\
\hline None & $1(1.1)$ \\
\hline TACE only & $48(51.6)$ \\
\hline TACE, RFA & $21(22.6)$ \\
\hline TACE, PEI & $4(4.3)$ \\
\hline TACE, RFA, PEI & $2(2.1)$ \\
\hline Resection & $1(1.1)$ \\
\hline Resection, TACE & $11(11.9)$ \\
\hline Resection, TACE, RFA & $2(2.1)$ \\
\hline Resection, TACE, PEI & $1(1.1)$ \\
\hline RFA & $2(2.1)$ \\
\hline \multicolumn{2}{|c|}{$\begin{array}{l}\text { Abbreviations: } \mathrm{HBV} \text {, hepatitis B virus; } \mathrm{HCV} \text {, hepatitis } \mathrm{C} \text { virus; SBRT, stereotactic body radiation therapy; TACE, transarterial chem } \\
\text { ablation; PEI, percutaneous ethanol injection. }\end{array}$} \\
\hline
\end{tabular}

Barcelona Clinic Liver Cancer guideline recommended ablative therapy as the curative treatment option for very early-stage HCC [16]. However, in some cases RFA cannot be safely and effectively performed, just like surgical resection. Therefore, there is an opportunity for SBRT to be performed on these selected HCC cases.

Here, we reported a very high local recurrence-free survival rate of $92.1 \%$ at 3 years. Many previous studies have reported 2 or 3 years local progression-free survival rates that range from $66.4 \%$ to $90 \%$ [5,6,17-19]. Although, these results cannot be directly compared due to the different inclusion criteria and prescribed doses, our results show that tumor ablation is possible using high dose of radiation and precision radiotherapy techniques. We assume that thorough IGRT is one of the most important ways to obtain a high local control rate over a long follow-up duration. In our series, most of patients were implanted with 3 gold seeds and pretreatment verification of the marker positions was conducted using 2 


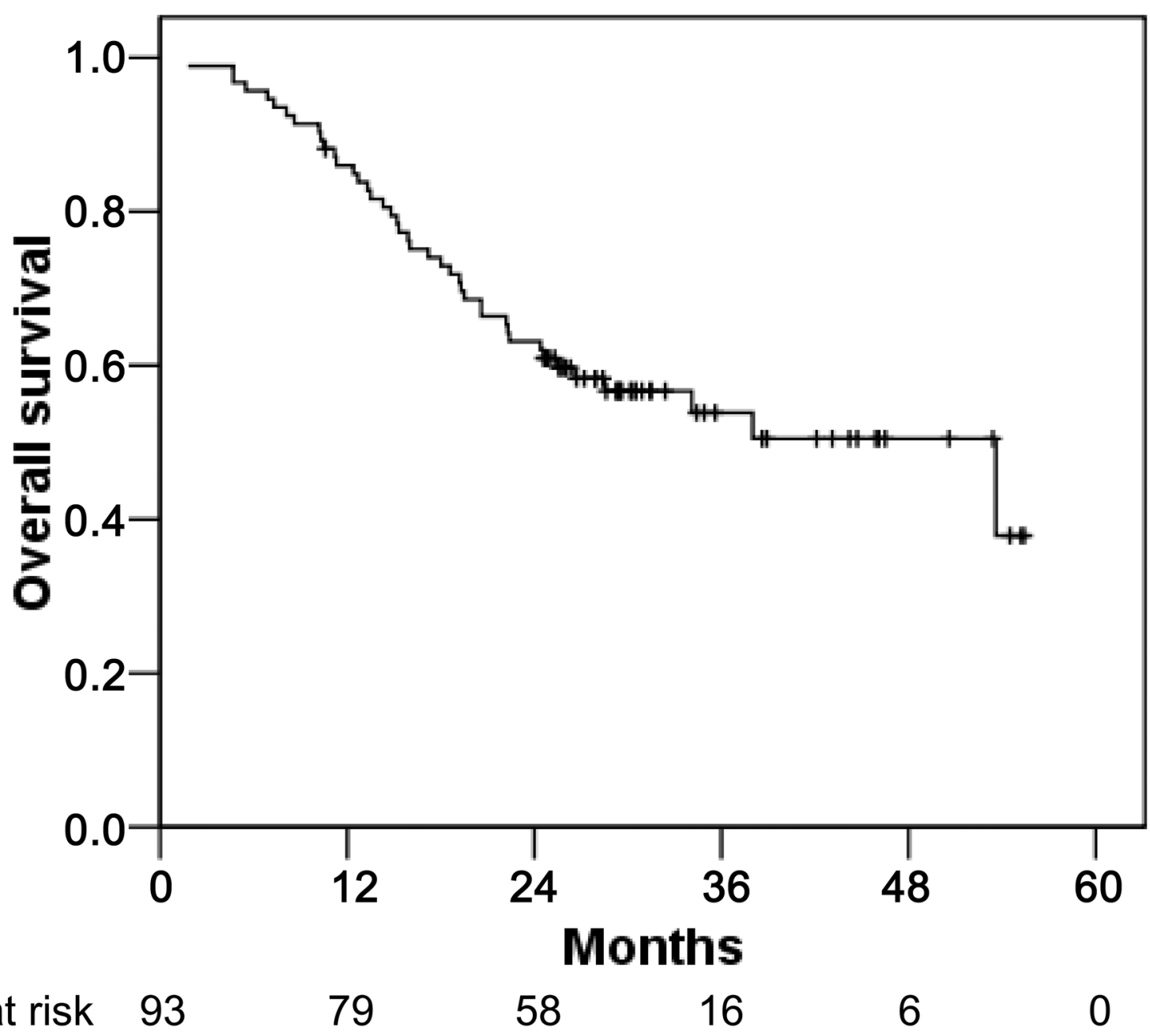

Figure 2. Overall survival rates of the enrolled patients. The 1 and 3 years survival rates are $86.0 \%$ and $53.8 \%$, respectively. doi: 10.1371/journal.pone.0079854.g002

Table 2. Response rates at 3 months after stereotactic body radiation therapy.

\begin{tabular}{|c|c|c|}
\hline & RECIST (version 1.1) & Modified RECIST \\
\hline & No. of lesions (\%) & No. of lesions (\%) \\
\hline Complete response & $16(15.5)$ & $53(51.5)$ \\
\hline Partial response & $47(45.7)$ & $22(21.4)$ \\
\hline Stable disease & $38(36.9)$ & $26(25.2)$ \\
\hline Not evaluated & $2(1.9)$ & $2(1.9)$ \\
\hline Response rate (CR+PR) & $63(61.2)$ & 75 (72.9) \\
\hline
\end{tabular}

Abbreviations: RECIST, Response Evaluation Criteria in Solid Tumors; CR, complete response; PR, partial response.

doi: 10.1371/journal.pone.0079854.t002

separate processes before the administration of each fraction to each patient. Even though pretreatment verifications are eagerly performed, there are still various uncertainties regarding to the respiration during treatment time. In consideration of our lower cumulative incidence of local failure, our PTV setup margin can cover the intrafractional respiratory uncertainties. In addition, a high local control rate may be associated with the inclusion of relatively small-sized tumors in this study.

In the present study cohort, the incidence of local recurrence was significantly related to tumor size. Although, there were some different size criteria compared with previously reported 
A

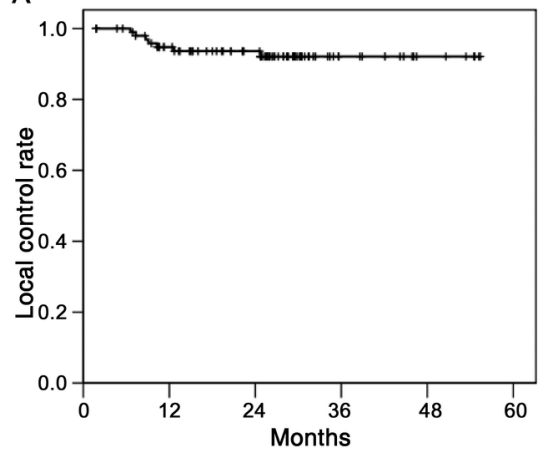

B

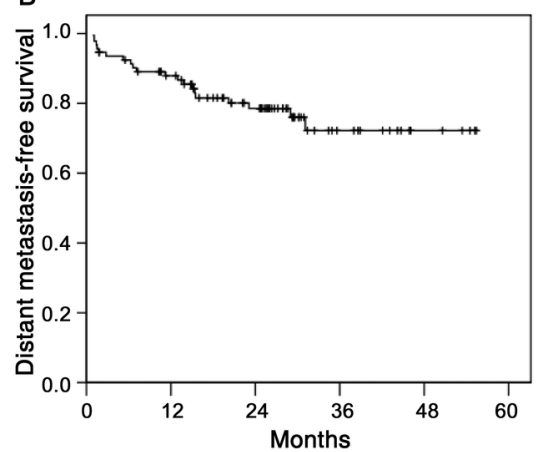

C

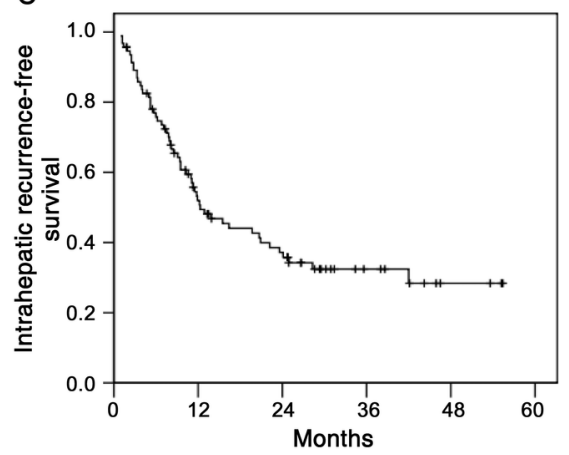

Figure 3. Local control and recurrence-free survival rates. (A) The local control rates at 1 and 3 years were $94.8 \%$ and $92.1 \%$, respectively. (B) Distant metastasis-free survival rates and (C) Intrahepatic recurrence-free survival rates following SBRT. doi: 10.1371/journal.pone.0079854.g003

results for SBRT, other authors have also reported a relationship between tumor size and the local control rate. Kwon et al. reported that patients with a tumor volume < $32 \mathrm{cc}$ demonstrate better in-field tumor responses and in-field progression-free survival rates than those with tumor volumes $\geq 32 \mathrm{cc}$ [6]. Andolino et al. have reported a local control rate at 2 years of $90 \%$, exceeding the local control rate of $65 \%$ at 1 year that was reported by Tse et al. They explained that the higher median total dose and higher median tumor volume contributed to the differences in the local control rates $[5,8]$. In other RFA series, very similar findings were observed, indicating that the local tumor control rate decreases as the tumor size increases [20,21]. All of these findings may be related to the prognostic significance of small-size HCCs. In a previous surgical series, it was revealed that large tumor size is a biological predictor of poor clinical prognosis, demonstrating a higher incidence of occult vascular invasion and advanced histological grade [22]. Therefore, care must be taken when treating $\mathrm{HCCs}>3 \mathrm{~cm}$, and prospective studies are needed to clarify the relationship between radiation dose escalation, target volume expansion, and the control of microscopic invasion. However, most HCC patients suffer from chronic liver disease, including liver cirrhosis, and have a limited functional normal liver volume. This may be an obstacle to performing studies on dose escalation because of the risks of hepatic toxicity following high-dose radiotherapy.

In our present analysis, the 3-year survival rate was $53.8 \%$ for patients with small, recurrent HCC. This result is comparable or slightly higher survival rates than that of other previously reported studies on SBRT $[5,6,17,19]$. Because SBRT was used as a salvage treatment following various locoregional therapies in most previously reported studies, it is difficult to compare the survival outcomes of SBRT, resection, and RFA when they are used as the initial treatment modalities. In the case of recurrent HCC following hepatectomy, repeated resection demonstrates slightly better survival outcomes than RFA, and the results of RFA are comparable to those of SBRT [23]. To the best of our knowledge, no standard treatment has been established for recurrent HCC; SBRT can be a good treatment option for patients with recurrent $\mathrm{HCC}$ and can achieve comparable survival outcomes to other aggressive salvage treatment options.

The primary failure pattern was intrahepatic recurrence and the 3-year intrahepatic recurrence-free survival rate was only $32.4 \%$. Although HCC recurrence rates vary across earlier studies, recurrence outside the radiation field is the main cause of failure in previously published reports on SBRT $[5,6,17]$. Recently, a very similar rate of intrahepatic recurrence was reported following the administration of salvage hypofractionated radiotherapy to patients with recurrent small HCC [24]. The authors suggested that intrahepatic recurrence might occur because patients received salvage radiotherapy at a more advanced disease state even if the recurrent tumor was small [24]. In our present study, most patients, except 1 individual who received SBRT as the initial treatment modality, were also treated with a median number of 5 courses (range 1-18) of locoregional therapies before SBRT, including resection, TACE, PEI, or RFA. Therefore, particular attention should be paid to interpreting the intrahepatic recurrence rates between studies. Moreover, in order to overcome the limitations of these clinical settings, trials are needed that assess the efficacy of various combinations of SBRT and other systemic treatment modalities including sorafenib.

Grade $\geq 3$ hepatic toxicities were observed in $6.5 \%$ of patients within 3 months after the SBRT and most patients showed improved or stable liver function without a serious hepatic damage during the follow-up period. This incidence may be acceptable, compared with that of the previous studies (range, 0-25.8\%) [5-8,17,18]. Among the patients who experienced the hepatic toxicities, one patient died due to the hepatic failure at 2 months after the treatment similar to those reported by Mendez Romero et al. [7]. However, the cause of this hepatic failure is difficult to pin down precisely. The hepatic function may be affected by various causes in patients with chronic liver disease, as we are all well aware, this event may be quite difficult to explain as what the real cause is. Although the risk factors for the hepatic damage were not found in the present study, the pretreatment Child-Pugh class is one of the important factors in predicting the hepatic toxicities according to the previous reports. Therefore, more attention could be 


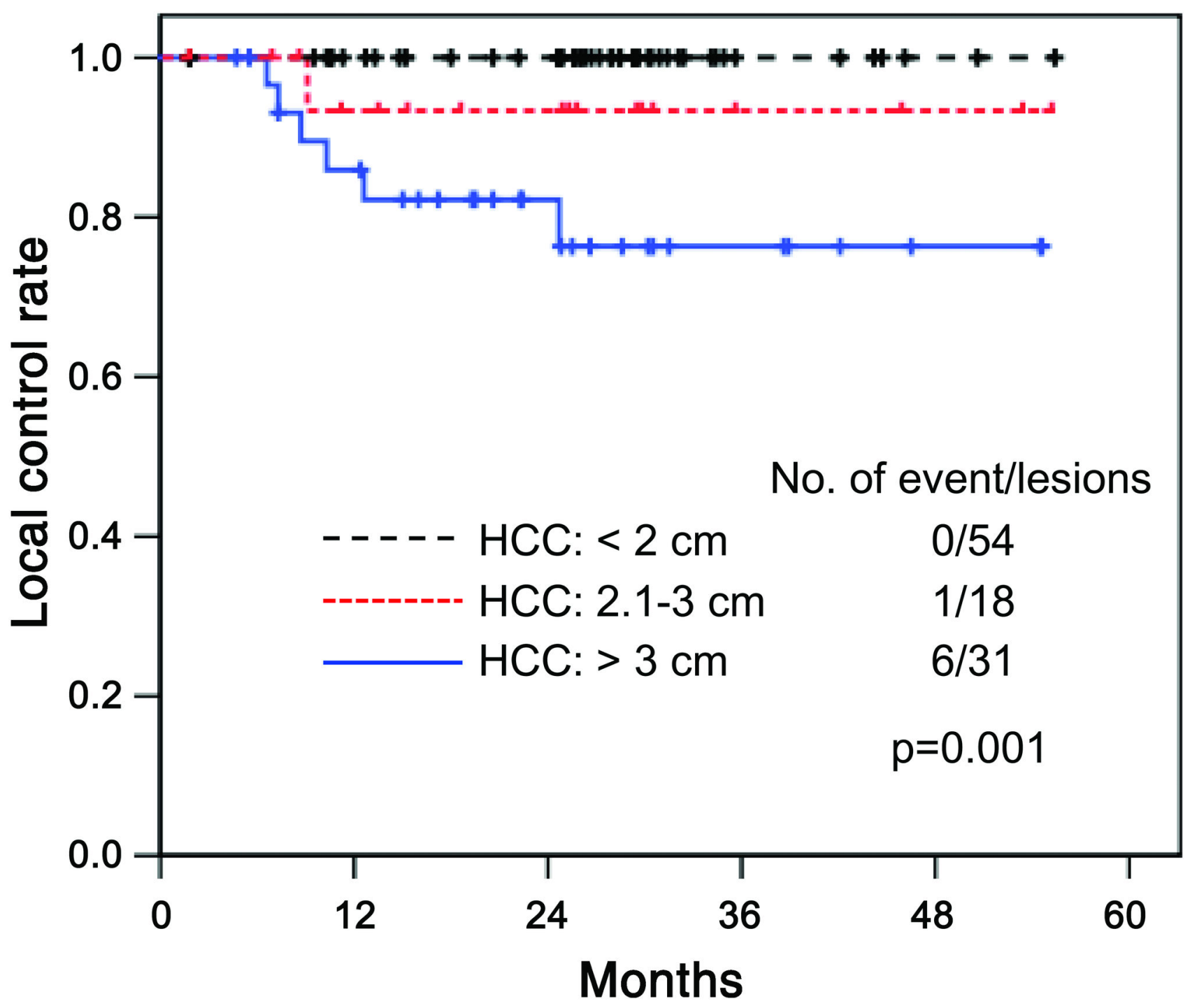

Figure 4. Analysis of local failures. Most local failures occurred in patients with $\mathrm{HCCs}>3 \mathrm{~cm}$, and the local control rate at 3 years was $76.3 \%$ in these cases, $93.3 \%$ in patients with $\mathrm{HCCs}$ between $2.1-3 \mathrm{~cm}$, and $100 \%$ in patients with $\mathrm{HCCs}<2 \mathrm{~cm}$, respectively $(p=0.001)$.

doi: 10.1371/journal.pone.0079854.g004

given in prescribing the total dose for patients with Child-Pugh $B$ cirrhosis $[5,7,25]$. Moreover, additional efforts aimed at finding the clinical and dose-volumetric parameters to predict the risk of hepatic toxicities in a large series of HCC patients treated with SBRT are needed.

In conclusion, the SBRT is a noninvasive and an excellent ablative treatment modality for patients with small, primary/ recurrent HCC. Our current results indicate that SBRT can be a good alternative modality for the treatment of small HCCs that are unsuitable for surgical resection or local ablative therapy. In order to accurately determine its efficacy and impact on overall survival compared with other local modalities, well-designed prospective investigations of SBRT are needed. In addition, further trials are also necessary to assess the efficacy of combinations of SBRT and other novel targeted agents for the treatment of patients with recurrent HCC. 
Table 3. Summary of the labortory finding and the radiation dose in patients who experienced the grade $\geq 3$ hepatic toxicities.

Case No. CTCAE grade Baseline liver function before SBRT

Dose $(\mathrm{Gy}) \mathrm{BED}\left(\mathrm{Gy}_{10}\right)$ Hightest level after SBRT $(\leq 3$ months) Normalized or stabilized C-P class AST (IU/L) ALT(IU/L) t-Bilirubin ( $\mathrm{mg} / \mathrm{dL})$

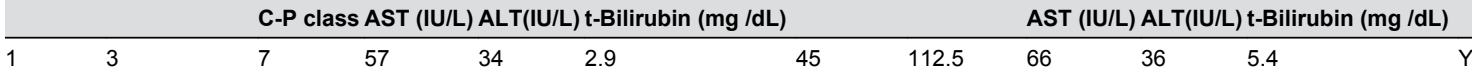

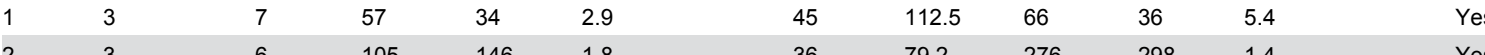

$2 \quad 3 \quad 6 \quad 105-146-1.8$

$\begin{array}{lllllllllllll}3 & 3 & 5 & 57 & 116 & 1.4 & 36 & 79.2 & 70 & 216 & 1.2 & \text { Yes }\end{array}$

$\begin{array}{llllllllllll}4 & 3 & 7 & 29 & 15 & 1.1 & 45 & 112.5 & 230 & 58 & 2.5 & \text { Yes }\end{array}$

$\begin{array}{llllllllllll}5 & 4 & 7 & 78 & 19 & 6.2 & 40 & 80 & 696 & 493 & 8.9 & \text { Yes }\end{array}$

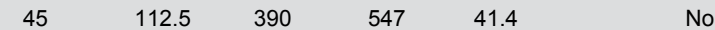

Abbreviations: CTCAE, the Common Terminology Criteria for Adverse Events; SBRT, Stereotactic Body Radiation Therapy; C-P class, Child-Pugh class; AST, aspartate aminotransferase; ALT, alanine aminotransferase; t-Bilirubin, total bilirubin; BED, biologically effective dose.

doi: 10.1371/journal.pone.0079854.t003 


\section{Author Contributions}

Conceived and designed the experiments: SMY YS. Lim HCL JP JHK. Performed the experiments: SMY YS. Lim MJP SYK
BC JHS KMK HCL YHC Yung Sang Lee SGL Yu Sun Lee JP. Analyzed the data: SMY MJP SYK BC Yu Sun Lee JP. Contributed reagents/materials/analysis tools: KMK YHC Yung Sang Lee SGL. Wrote the manuscript: SMY YS. Lim JHK.

\section{References}

1. Ferlay J, Shin HR, Bray F, Forman D, Mathers C et al. (2010) Estimates of worldwide burden of cancer in 2008: GLOBOCAN 2008 Int J Cancer 127: 2893-2917. doi:10.1002/ijc.25516. PubMed: 21351269.

2. Bruix J, Sherman M (2011) Management of hepatocellular carcinoma: an update. Hepatology 53: 1020-1022. doi:10.1002/hep.24199. PubMed: 21374666

3. Lau WY, Lai EC (2007) Salvage surgery following downstaging of unresectable hepatocellular carcinoma--a strategy to increase resectability. Ann Surg Oncol 14: 3301-3309. doi:10.1245/ s10434-007-9549-7. PubMed: 17891443.

4. Krishnan S, Dawson LA, Seong J, Akine Y, Beddar S et al. (2008) Radiotherapy for hepatocellular carcinoma: an overview. Ann Surg Oncol 15: 1015-1024. doi:10.1245/s10434-007-9729-5. PubMed: 18236114.

5. Andolino DL, Johnson CS, Maluccio M, Kwo P, Tector AJ et al. (2011) Stereotactic body radiotherapy for primary hepatocellular carcinoma. Int J Radiat Oncol Biol Phys 81: e447-e453. doi:10.1016/j.jirobp. 2011.04.011. PubMed: 21645977.

6. Kwon JH, Bae SH, Kim JY, Choi BO, Jang HS et al. (2010) Long-term effect of stereotactic body radiation therapy for primary hepatocellular carcinoma ineligible for local ablation therapy or surgical resection. Stereotactic radiotherapy for liver cancer. BMC Cancer 10: 475. doi: 10.1186/1471-2407-10-475. PubMed: 20813065

7. Méndez Romero A, Wunderink W, Hussain SM, De Pooter JA, Heijmen BJ et al. (2006) Stereotactic body radiation therapy for primary and metastatic liver tumors: A single institution phase i-ii study. Acta Oncol 45: 831-837. doi:10.1080/02841860600897934. PubMed: 16982547.

8. Tse RV, Hawkins M, Lockwood G, Kim JJ, Cummings B et al. (2008) Phase I study of individualized stereotactic body radiotherapy for hepatocellular carcinoma and intrahepatic cholangiocarcinoma. J Clin Oncol 26: 657-664. doi:10.1200/JCO.2007.14.3529. PubMed: 18172187.

9. Schefter TE, Kavanagh BD, Timmerman RD, Cardenes HR, Baron A et al. (2005) A phase I trial of stereotactic body radiation therapy (SBRT) for liver metastases. Int J Radiat Oncol Biol Phys 62: 1371-1378. doi: 10.1016/j.jirobp.2005.01.002. PubMed: 16029795.

10. Pan CC, Kavanagh BD, Dawson LA, Li XA, Das SK et al. (2010) Radiation-associated liver injury. Int J Radiat Oncol Biol Phys 76: S94-100. doi:10.1016/j.jijrobp.2009.06.092. PubMed: 20171524

11. Ishii H, Furuse J, Kinoshita T, Konishi M, Nakagohri T et al. (2008) Hepatectomy for hepatocellular carcinoma patients who meet the Milan criteria. Hepatogastroenterology 55: 621-626. PubMed: 18613420.

12. Chen MS, Li JQ, Zheng Y, Guo RP, Liang HH et al. (2006) A prospective randomized trial comparing percutaneous local ablative therapy and partial hepatectomy for small hepatocellular carcinoma. Ann Surg 243: 321-328. doi:10.1097/01.sla.0000201480.65519.b8. PubMed: 16495695.

13. Feng K, Yan J, Li X, Xia F, Ma K et al. (2012) A randomized controlled trial of radiofrequency ablation and surgical resection in the treatment of small hepatocellular carcinoma. J Hepatol 57: 794-802. doi:10.1016/ j.jhep.2012.05.007. PubMed: 22634125.

14. Huang J, Yan L, Cheng Z, Wu H, Du L et al. (2010) A randomized trial comparing radiofrequency ablation and surgical resection for HCC conforming to the Milan criteria. Ann Surg 252: 903-912. doi:10.1097/ SLA.0b013e3181efc656. PubMed: 21107100.

15. Lencioni R (2010) Loco-regional treatment of hepatocellular carcinoma. Hepatology 52: 762-773. doi:10.1002/hep.23725. PubMed: 20564355.

16. Forner A, Llovet JM, Bruix J (2012) Hepatocellular carcinoma. Lancet 379: 1245-1255. doi:10.1016/S0140-6736(11)61347-0. PubMed: 22353262.

17. Huang WY, Jen YM, Lee MS, Chang LP, Chen CM et al. (2012) Stereotactic body radiation therapy in recurrent hepatocellular carcinoma. Int J Radiat Oncol Biol Phys 84: 355-361. doi:10.1016/ j.ijrobp.2011.11.058. PubMed: 22342300.

18. Seo YS, Kim MS, Yoo SY, Cho CK, Choi CW et al. (2010) Preliminary result of stereotactic body radiotherapy as a local salvage treatment for inoperable hepatocellular carcinoma. J Surg Oncol 102: 209-214. doi: 10.1002/jso.21593. PubMed: 20740576.

19. Taguchi H, Sakuhara $Y$, Hige S, Kitamura K, Osaka $Y$ et al. (2007) Intercepting radiotherapy using a real-time tumor-tracking radiotherapy system for highly selected patients with hepatocellular carcinoma unresec table with other modalities. Int J Radiat Oncol Biol Phys 69: 376-380. doi:10.1016/j.ijrobp.2007.07.1483. PubMed: 17869660.

20. Livraghi T, Lazzaroni S, Meloni F (2001) Radiofrequency thermal ablation of hepatocellular carcinoma. Eur $\mathrm{J}$ Ultrasound 13: 159-166. doi:10.1016/S0929-8266(01)00128-8. PubMed: 11369527.

21. Sala M, Llovet JM, Vilana R, Bianchi L, Solé M et al. (2004) Initial response to percutaneous ablation predicts survival in patients with hepatocellular carcinoma. Hepatology 40: 1352-1360. doi:10.1002/hep. 20465. PubMed: 15565564.

22. Pawlik TM, Delman KA, Vauthey JN, Nagorney DM, Ng IO et al. (2005) Tumor size predicts vascular invasion and histologic grade: Implications for selection of surgical treatment for hepatocellular carcinoma. Liver Transpl 11: 1086-1092. doi:10.1002/lt.20472. PubMed: 16123959.

23. Yang $W$, Chen $M H$, Yin SS, Yan K, Gao W et al. (2006) Radiofrequency ablation of recurrent hepatocellular carcinoma after hepatectomy: therapeutic efficacy on early- and late-phase recurrence. AJR Am J Roentgenol 186: S275-S283. doi:10.2214/AJR.04.1573. PubMed: 16632688.

24. Bae SH, Park HC, Lim do H, Lee JA, Gwak GY et al. (2012) Salvage treatment with hypofractionated radiotherapy in patients with recurrent small hepatocellular carcinoma. Int J Radiat Oncol Biol Phys 82: e603e607. doi:10.1016/j.ijrobp.2011.09.053. PubMed: 22208963.

25. Cárdenes HR, Price TR, Perkins SM, Maluccio M, Kwo P et al. (2010) Phase I feasibility trial of stereotactic body radiation therapy for primary hepatocellular carcinoma. Clin Transl Oncol 12: 218-225. doi:10.1007/ s12094-010-0492-x. PubMed: 20231127. 